\title{
Effects of secondary infections on the multidrug-resistant Tuberculosis: A cohort study
}

\author{
Nurlan Tabriz ${ }^{1} \mathbb{D}$, Zhanara B. Nurtazina ${ }^{1}$, Margulan T. Kozhamuratov ${ }^{1}$, Kuliya Skak $^{1}$, Zhumat Mutaikhan $^{1}$ \\ Received: 21 Dec 2020 \\ Published: 17 Aug 2021
}

\begin{abstract}
Background: Tuberculosis (TB) causes over a million deaths annually and is still one of the most important public health problems worldwide. According to the World Health Organization estimates, the highest rates of TB in the European Region are in Tajikistan, Kazakhstan, Moldova, Kyrgyzstan, Romania, and Uzbekistan. The purpose of this study was to investigate the spectrum of nonspecific microorganisms isolated in patients with multidrug-resistant $\mathrm{TB}$ in Central Kazakhstan and to assess their susceptibility to antimicrobial drugs.

Methods: The patients were divided into 2 groups: group 1 with multidrug-resistant forms of pulmonary TB ( $\mathrm{n}=107$ patients); group 2 with sensitive forms of pulmonary TB $(n=122$ patients). Gender, age, and social status of the patients were studied. Microorganisms were identified using the MALDI-TOF method. The statistical significance of different values for binary and nominal parameters was determined using the chi-square test. Changes in binary variables were analyzed using the McNeimer test.

Results: During the study, an expectedly high proportion of tetracycline-resistant pneumococcal strains $(66.7 \%$ and $60 \%$, respectively) was isolated, which was a consequence of a long-term and practically uncontrolled use of these drugs in Kazakhstan. Fluoroquinolones showed low activity. The results showed that beta-lactam antibacterial drugs maintained their high activity against the causative agents of pneumococcal infection.

Conclusion: It was concluded that secondary microorganisms isolated in patients with multidrug-resistant TB were represented by the strains that were resistant to modern antibacterial drugs. Therefore, for appropriate antibiotic prescription, it is necessary to study materials from the respiratory system in all patients admitted for TB treatment to study the spectrum of nonspecific microorganisms and assess their susceptibility to antimicrobial drugs.
\end{abstract}

Keywords: Health care, Treatment efficacy, Non-specific microorganisms, Antibacterial drugs

Conflicts of Interest: None declared

Funding: None

*This work has been published under CC BY-NC-SA 1.0 license.

Copyright $\odot$ Iran University of Medical Sciences

Cite this article as: Tabriz N, Nurtazina ZB, Kozhamuratov MT, Skak K, Mutaikhan Z. Effects of secondary infections on the multidrug-resistant Tuberculosis: A cohort study. Med J Islam Repub Iran. 2021 (17 Aug);35:105. https://doi.org/10.47176/mjiri.35.105

\section{Introduction}

Tuberculosis (TB) causes over a million deaths annually

Corresponding author: Dr Nurlan Tabriz, n.tabriz5921@ubogazici.in

1. Department of Infectious Diseases and Phthisiatrics, Karaganda Medical University, Karaganda, Republic of Kazakhstan and is still one of the most important public health prob-

$\uparrow$ What is "already known" in this topic:

Although patients with pulmonary TB receive long courses of antibiotic therapy (streptomycin, rifampicin, etc), they often develop secondary infections commonly caused by opportunistic microorganisms (OMs) resistant to these drugs. OMs create favorable conditions for the spread of mycobacteria and new lesions.

\section{$\rightarrow$ What this article adds:}

Secondary microorganisms isolated in patients with multidrugresistant $\mathrm{TB}$ are represented by the strains that are resistant to modern antibacterial drugs, and for appropriate antibiotic prescription, it is necessary to study materials from the respiratory organs in all patients admitted for TB treatment to obtain the spectrum of nonspecific microorganisms and assess their sensitivity to antimicrobial drugs. 
lems worldwide. According to the World Health Organization estimates, the highest rates of TB in the European Region are in Tajikistan, Kazakhstan, Moldova, Kyrgyzstan, Romania, and Uzbekistan (1-3). Kazakhstan is one of 18 countries in the European Region with high rates of multidrug-resistant TB (MDR-TB). Despite active measures for the diagnosi $\mathrm{s}$ and treatment of $\mathrm{TB}$ in $\mathrm{Ka}-$ zakhstan, the rates remain high (3). The problem of multidrug resistance poses a great problem for TB treatment and remains the subject of thorough monitoring by global health care (4-7). A steady increase in the spread of resistant Mycobacterium TB strains is of particular concern, including the most dangerous strains with extensive and multidrug resistance (XDR-TB and MDR-TB, respectively). Multidrug-resistant TB is one of the main factors resulting in an increased mortality and low efficacy of treatment (8-12).

The course of TB is often complicated with a nonspecific inflammation, which changes both clinical manifestations of TB and the course and outcome of the disease (13). Despite the fact that patients with pulmonary TB receive long courses of antibiotic therapy (streptomycin, rifampicin, etc.), they often develop secondary infections commonly caused by opportunistic microorganisms (OMs) resistant to these drugs (14). Antibacterial chemotherapy, which has prolonged the life of patients with TB, has increased the importance of OMs as etiological agents of pyoinflammatory diseases of the lower respiratory tract (LRT) in patients with pulmonary TB. The airways of patients with pulmonary TB are nonsterile. OMs often colonize them even during remission, as the level of colonization resistance in such patients is lower. Causing a pathological process in the lungs, OMs aggravate the course of the underlying disease. OMs create favorable conditions for the spread of mycobacteria and new lesions.

The combination of infectious pathogens (15-17) has negative effects on the prognosis and the possibility of clinical recovery $(18,19)$. Secondary infections, occurring in the form of exacerbation of nonspecific bronchitis and pneumonia and complicating the course of fibrouscavernous TB, infiltrative TB, TB, et cetera, are diverse and are not caused by a specific pathogen $(20,21)$. The rates of combination of $\mathrm{TB}$ with nonspecific respiratory diseases and other non-TBinfections vary from $7 \%$ to $49 \%$ (16-19). In patients with recurrent pulmonary $\mathrm{TB}$, chronic nonspecific respiratory diseases occur in $17.5-63.2 \%$ of cases $(22,23)$. The combination of TB with nonspecific pulmonary diseases significantly complicates the course of TB, causing multiple symptoms, predominance of alterative and exudative changes. Combined pathologies result in the worst treatment results in pulmonary $\mathrm{TB}$, decreased rates of cavity closure and sputum abacillation, and longer treatment duration $(24,25)$.

Timely diagnosis of secondary LRT infections in patients with pulmonary TB and targeted treatment of complications is one of the urgent problems of modern phthisiology (14). Treatment of both TB and concomitant diseases caused by nonspecific microorganisms requires the use of broad-spectrum antibiotics together with antiTB drugs. Treatment of nosocomial infections is a diffi- cult process, as nosocomial microorganisms have a different spectrum of susceptibility to antibacterial drugs than community-acquired pathogenis. The emergence and administration of new generations of antibiotics result primarily in qualitative changes in nosocomial flora (26). Accumulated data on antibiotic resistance of secondary microorganisms in pulmonary TB show the need to study the spectrum of isolated microorganisms and their sensitivity to antimicrobial drugs $(27,28)$.

The purpose of this study was to investigate the spectrum of nonspecific microorganisms isolated in patients with multidrug-resistant TB in Central Kazakhstan and to assess their susceptibility to antimicrobial drugs.

\section{Methods}

A cohort study was performed at the microbiological laboratory of the Medical University of Karaganda. The strains of nonspecific microorganisms were obtained from sputum samples from the patients with a confirmed diagnosis of TB. The results of microbiological examination of sputum obtained from 229 TB patients admitted for treatment to inpatient departments of the Regional TB Dispensary in Karaganda in 2014-2015 and 2018-2019 were analyzed. To achieve the purpose of the study and assess the studied (quantitative) parameters, depending on the isolation method, the patients were divided into 2 groups: group 1 with multidrug-resistant forms of pulmonary TB ( $n=107$ patients); group 2 with sensitive forms of pulmonary TB ( $\mathrm{n}=122$ patients). To study the spectrum of nonspecific microorganisms isolated in the patients with MDR-TB and assess their susceptibility to antimicrobial drugs, we studied gender, age, and social status of the patients, analyzed cases by type, clinical structure of TB, extent of the process, and destructive changes in the lungs.

The studied materials were collected into sterile containers and delivered to the microbiological laboratory no later than 2 hours after sputum collection. Inoculation of nonspecific microorganisms was done in culture media with the isolation of pure cultures. Microorganisms were identified using the MALDI-TOF method with the Microflex mass spectrometer (Bruker Daltonics). During the MALDI-TOF, a double (duplicate) application of the culture with identification according to the standard protocol recommended by Bruker Daltonics was used. Mass spectra were calibrated using Escherichia coli ribosomal proteins (bacterial standard). Protein spectra were analyzed using the MALDI Biotyper (Version 3, Bruker Daltonics).

The results were expressed as a score from 0 to 3 . A score $>1.7$ corresponded to a high degree of reliability of identification up to genus, and a score $>2.0$ corresponded to reliable identification up to species. At the same time, a score $>1.7$ was considered as the minimum value of the score coefficient required for species identification. At a score $<1.7$, the identification result was considered invalid and the test was repeated (28). To determine the susceptibility of microorganisms to antibiotics, a disc diffusion method was used, according to the recommendations of the Clinical and Laboratory Standards Institute (CLSI 2012) (29). Resistance to methicillin (oxacillin) and other $\beta$-lactam antibiotics of S.aureus isolates was determined 
based on the resistance to oxacillin and cefoxitin (30). The extended $\beta$-lactamase spectrum in gram-negative bacteria was determined using phenotypic methods (31).

The 2 groups were compared on a quantitative scale using the nonparametric Mann-Whitney $U$ test. The relative attribute rates in different independent populations were compared using a $\mathrm{z}$ test. The statistical significance of different values for binary and nominal parameters was determined using the chi-square test. Changes in binary variables were analyzed using the McNemar test. To describe quantitative parameters, the means and standard deviations were used in the "M $\pm \mathrm{S}$ " format. The level of statistical significance was accepted as an error probability level of .05. Statistical data processing was performed using the Statistica 10 and SAS JMP 11 software packages.

\section{Results}

Gender characteristics of the patients showed the prevalence of men in both groups: $84(78.5 \%)$ in group 1 and 85 $(69.7 \%)$ in group 2; and also, $23(21.5 \%)$ and 37 (30.3\%) women in groups 1 and 2, respectively. There were no significant differences between the groups, $p>0.005$. The analysis of the age of the patients showed that the groups did not differ in age, $\mathrm{p}>0.005$ (Table 1 ).

The analysis of patients by case type is shown in Table 2. The development of multidrug resistance is caused by ineffective treatment, noncompliance during previous treatment, and accordingly disease recurrence.

Table 3 shows patient distribution by diagnosis of tuberculous process. Infiltrative tuberculosis was most common in both groups.

A history of nonspecific pulmonary diseases was observed in $16(15.0 \%)$ patients in group 1 and $22(18.0 \%)$ patients in group $2(\mathrm{p}>0.005)$. Another TB comorbidity, HIV infection, was observed in $5(4.7 \%)$ patients in group 1 and $7(5.7 \%)$ patients from Group 2 ( $p>0.005)$. Before therapy, acid-fast mycobacteria in sputum was detected using bacterioscopy in $46(43.0 \%)$ patients in group 1 and in $59(48.4 \%)$ patients in group 2; there were no significant differences between the groups $(\mathrm{p}>0.005)$. Therefore, the characteristics of the compared groups showed that multidrug resistance affected the distribution of clinical forms, as evidenced by the prevalence of common destructive forms of TB with a severe progressive course and predominant and massive isolation of mycobacterium TB. The above-mentioned unfavorable factors could influence the structure and sensitivity of the secondary nonspecific flora. The comparison groups did not differ in gender, age, or bacteria isolation.

The study included 229 strains isolated in patients with respiratory infections of various localization who were admitted to the Regional TB Dispensary in Karaganda; 218 strains were isolated from sputum, 5 from pleural fluid, and 6 from throat swabs. The spectrum of isolated microorganisms is presented in Table 4 in descending

Table 1. Age Distribution of the Patients

\begin{tabular}{lcccc}
\hline & \multicolumn{3}{c}{ Group 1 (n=107) } & Group 2(n=122) \\
\cline { 2 - 5 } & $\mathrm{N}$ & $\mathrm{P} \%$ & $\mathrm{~N}$ & $\mathrm{P}$ \\
\hline Under 20 & 3 & 2.8 & 16 & 4.92 \\
$21-30$ & 14 & 13.08 & 23 & 13.11 \\
$31-40$ & 30 & 28.04 & 31 & 18.85 \\
$41-50$ & 30 & 28.04 & 23 & 25.41 \\
$51-60$ & 18 & 16.82 & 23 & 18.85 \\
Over 61 & 12 & 11.21 & 18.85 \\
\hline
\end{tabular}

Table 2. Case Type Distribution

\begin{tabular}{lcccc}
\hline & \multicolumn{2}{c}{ Group 1(n=107) } & Group 2 (n=122) \\
\cline { 2 - 5 } & $\mathrm{N}$ & $\mathrm{P} \%$ & $\mathrm{~N}$ & $\mathrm{P} \%$ \\
\hline New case & 48 & 44.86 & 3 & 79.51 \\
Treatment failure & 13 & 12.15 & 2.46 & 17 \\
Relapse & 33 & 30.84 & 3 & 13.93 \\
Treatment after discontinuation & 9 & 8.41 & 1 & 0.46 \\
Switched & 2 & 1.87 & 1 & 0.82 \\
Other & 1 & 0.93 & 0.00 \\
Noncompliance & 1 & 0.93 & \\
\hline
\end{tabular}

Table 3. Distribution by Diagnosis of Tuberculous Process

\begin{tabular}{|c|c|c|c|c|}
\hline & \multicolumn{2}{|c|}{ Group $1(n=107)$} & \multicolumn{2}{|c|}{ Group $2(n=122)$} \\
\hline & $\mathrm{n}$ & $\mathrm{P} \%$ & $\mathrm{~N}$ & $\mathrm{P} \%$ \\
\hline Disseminated tuberculosis & 4 & 3.74 & 12 & 9.84 \\
\hline Focal tuberculosis & 1 & 0.93 & 2 & 1.64 \\
\hline Infiltrative tuberculosis & 68 & 63.55 & 76 & 62.3 \\
\hline Caseous pneumonia & 4 & 3.74 & & 0 \\
\hline Tuberculoma & 8 & 7.48 & 11 & 9.02 \\
\hline Cavernous tuberculosis & 1 & 0.93 & 1 & 0.82 \\
\hline Fibrous-cavernous tuberculosis & 17 & 15.89 & 4 & 3.28 \\
\hline Tuberculous pleuritis & 1 & 0.93 & 6 & 4.92 \\
\hline Miliary tuberculosis & - & & 1 & 0.82 \\
\hline $\begin{array}{l}\text { Extrapulmonary forms of tuberculosis combined with nonspecific } \\
\text { respiratory diseases }\end{array}$ & 2 & 1.87 & 9 & 7.38 \\
\hline
\end{tabular}


order of detection rates. The analysis of species showed a predominance of Escherichia coli in group $1(\mathrm{n}=27$; $25.2 \%)$ and in group $2(\mathrm{n}=39 ; 32.0 \%)$, Staphylococcus aureus in group $1(\mathrm{n}=15 ; 14 \%)$ and in group $2(\mathrm{n}=10$; $8.2 \%)$, Klebsiella pneumoniae in group $1(\mathrm{n}=14 ; 13.1 \%)$ and in group $2(31 ; 25.4 \%)$, and Pseudomonas aeruginosa in group $1(\mathrm{n}=13 ; 12.1 \%)$ and in group $2(1.8 ; 6.6 \%)$.

Following the isolation of nonspecific flora, the susceptibility of the isolated pathogens to antibacterial drugs was determined during further microbiological tests. The results of this analysis are shown in Table 5, where antibiotics are categorized.

Glycopeptides (Vancomycin, Teicoplanin) and oxazolidinones (Linezolid) appeared to be the most effective in vitro against pathogens isolated from pathological materials. In our study, resistance to these drugs was not observed, which is consistent with the reresults of similar Russian and Kazakh studies $(32,33)$. Microorganisms in both groups were equally more susceptible to carbapenems (Imipenem, Meropenem, Doripenem), $87 \%$ and $94.5 \%$, respectively; chloramphenicol, $86.2 \%$ and $88.8 \%$, respectively;

Table 4. Results of microbiological studies of secondary microorganisms in patients with Tuberculosis

\begin{tabular}{|c|c|c|c|c|}
\hline & \multicolumn{2}{|c|}{ Group $1(n=107)$} & \multicolumn{2}{|c|}{ Group $2(n=122)$} \\
\hline & $\mathrm{N}$ & $\mathrm{p} \%$ & $\mathrm{~N}$ & $\mathrm{P} \%$ \\
\hline Escherichia coli & 27 & 25.23 & 39 & 31.97 \\
\hline Staphylococcus aureus & 15 & 14.02 & 10 & 8.20 \\
\hline Klepsiella pneumonia & 14 & 13.08 & 31 & 25.41 \\
\hline Pseudomonas aeruginosa & 13 & 12.15 & 8 & 6.56 \\
\hline Klepsiella oxytoca & 6 & 5.61 & 7 & 5.74 \\
\hline Streptococcus pneumonia & 6 & 5.61 & 5 & 4.10 \\
\hline Acinetobacter baumann 2 & 5 & 4.67 & 5 & 4.10 \\
\hline Enterobacter cloacae & 5 & 4.67 & 2 & 1.64 \\
\hline Serratia marcescens & 3 & 2.80 & 4 & 3.28 \\
\hline Enterobacter aerogenes & 3 & 2.80 & 1 & 0.82 \\
\hline Enterococcus faecalis & 2 & 1.87 & 3 & 2.46 \\
\hline Stenotrophomanas maltrophilia & 2 & 1.87 & 0 & 0.00 \\
\hline Streptococcus mitis & 1 & 0.93 & 0 & 0.00 \\
\hline Proteus mirabilis & 1 & 0.93 & 1 & 0.82 \\
\hline Enterobacter sp. & 0 & 0.00 & 2 & 1.64 \\
\hline Staphylococcus saprophyticus & 1 & 0.93 & 0 & 0.00 \\
\hline Streptococcus sanguis & 1 & 0.93 & 0 & 0.00 \\
\hline Acinetobacter jun2 & 1 & 0.93 & 0 & 0.00 \\
\hline Citrobacter freund 2 & 0 & 0.00 & 2 & 1.64 \\
\hline Streptococcus anginosus & 1 & 0.93 & 0 & 0.00 \\
\hline Enterococcus faecium & 0 & 0.00 & 1 & 0.82 \\
\hline Klebsiella sp. & 0 & 0.00 & 1 & 0.82 \\
\hline Pseudomonas alcaligenes & 0 & 0.00 & 1 & 0.82 \\
\hline
\end{tabular}

First generation aminoglycosides (Kanamycin, Neomycin, Streptomycin)

Second generation aminoglycosides (Netilmicin, Gentamicin, Tobramycin, Sisomicin)

Third generation aminoglycosides (Amikacin, Isepamicin)

First generation fluoroquinolones Nalidixid acid

Second generation fluoroquinolones (Ciprofloxacin, Ofloxacin, Norfloxacin)

Third generation fluoroquinolones (Levofloxacin)

\begin{tabular}{|c|c|c|c|c|c|c|c|c|c|c|}
\hline 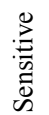 & 胥 & 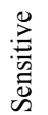 & 吾 & $\frac{\partial^{0}}{2}$ & $\begin{array}{l}0 \\
\curvearrowleft \\
= \\
=1 \\
=\end{array}$ & $\begin{array}{l}\Xi \\
\curvearrowleft \\
\vdots \\
\pm \\
=\end{array}$ & 을 & $\begin{array}{l}\circlearrowright \\
\curvearrowleft \\
=1 \\
=1\end{array}$ & $\begin{array}{l}\circlearrowright \\
\curvearrowleft \\
\vdots \\
\vdots\end{array}$ & $\mathrm{N}$ \\
\hline
\end{tabular}

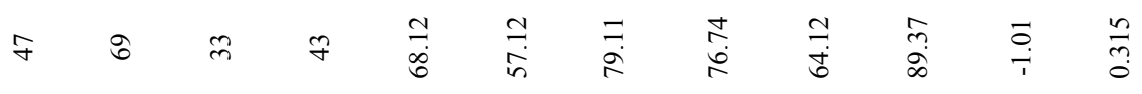

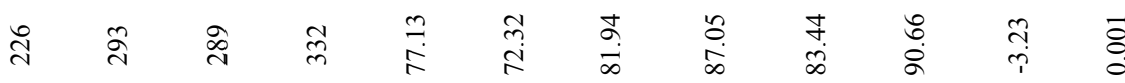

\begin{tabular}{|c|c|c|c|c|c|c|c|c|c|}
\hline$\infty$ & $a_{1}$ & ๙ & $\underset{+}{\nabla}$ & 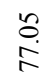 & $\begin{array}{l}0 \\
\text { ñ }\end{array}$ & $\begin{array}{l}\dot{\sigma} \\
\text { ๙े }\end{array}$ & 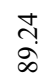 & $\begin{array}{l}\text { J } \\
\infty \\
\alpha\end{array}$ & 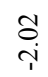 \\
\hline
\end{tabular}

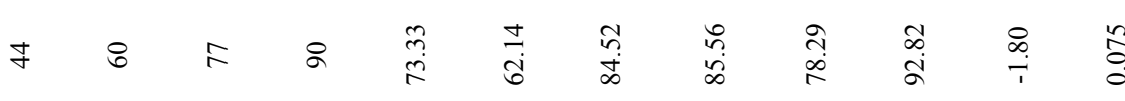

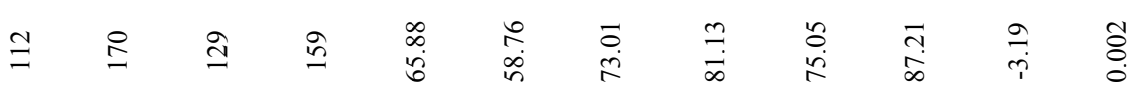

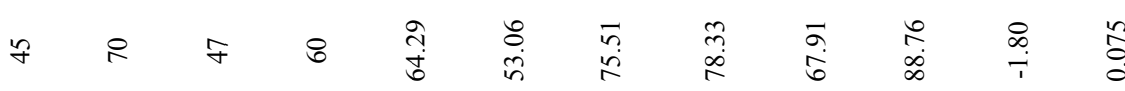




\begin{tabular}{|c|c|c|c|c|c|c|c|c|c|c|c|c|}
\hline & \multicolumn{6}{|c|}{ Group 1} & \multicolumn{4}{|c|}{ Group 2} & \multirow[b]{2}{*}{$\mathrm{N}$} & \multirow[b]{2}{*}{ 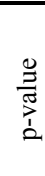 } \\
\hline & 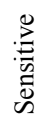 & $\stackrel{\Xi \pi}{0}$ & : & 胥 & $\frac{0^{\circ}}{2}$ & $\begin{array}{l}\circlearrowright \\
ٌ \\
\vdots \\
=1\end{array}$ & $\begin{array}{l}j \\
\vdots \\
\vdots \\
\vdots \\
\vdots\end{array}$ & 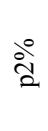 & $\begin{array}{l}\bar{D} \\
\vdots \\
\vdots \\
\vdots \\
=1\end{array}$ & 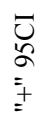 & & \\
\hline $\begin{array}{l}\text { First generation cephalosporins } \\
\text { (Cefazolin) }\end{array}$ & N & n & m & m & $\begin{array}{l}\hat{\sigma} \\
: \\
8\end{array}$ & $\stackrel{m}{m}$ & 完 & 8 & 8 & 8 & $\underset{T}{\stackrel{1}{\top}}$ & $\begin{array}{l}\infty \\
\infty \\
\\
0\end{array}$ \\
\hline $\begin{array}{l}\text { Second generation cephalosporins } \\
\text { (Cefuroxime, Cefamandole, Cefoxitin) }\end{array}$ & $\mathscr{F}$ & in & $\approx$ & $\stackrel{\infty}{\sim}$ & $\begin{array}{l}\infty \\
\infty \\
i \\
i\end{array}$ & $\stackrel{4}{6}$ & $\begin{array}{l}\hat{\imath} \\
\dot{\infty}\end{array}$ & $\underset{\infty}{\stackrel{\infty}{\infty}}$ & $\begin{array}{l}\stackrel{2}{2} \\
\stackrel{2}{2}\end{array}$ & $\begin{array}{l}8 \\
\dot{0} \\
\dot{+}\end{array}$ & ș & $\underset{0}{ت}$ \\
\hline $\begin{array}{l}\text { Third generation cephalosporins } \\
\text { (Cefotaxime, Ceftriaxone, Ceftazidime, } \\
\text { Cefixime, Cefoperazone, Cefpodoxime) }\end{array}$ & 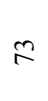 & $\Xi$ & $\tilde{\varrho}$ & $\underset{n}{-2}$ & $\begin{array}{l}\dot{J} \\
\dot{J}\end{array}$ & $\underset{n}{n}$ & 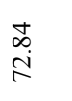 & $\frac{i}{i}$ & $\begin{array}{l}n \\
n \\
\infty\end{array}$ & $\underset{\infty}{\stackrel{5}{i}}$ & 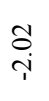 & $\stackrel{?}{\stackrel{2}{0}}$ \\
\hline $\begin{array}{l}\text { Fourth generation cephalosporins } \\
\text { (Cefepime) }\end{array}$ & $\stackrel{n}{n}$ & $\hat{\sigma}$ & $\infty$ & J & $\begin{array}{l}\hat{\sigma} \\
\dot{J}\end{array}$ & $\begin{array}{l}\vec{ָ} \\
\text { ț }\end{array}$ & $\begin{array}{l}\hat{\sigma} \\
\ddot{\infty} \\
\infty\end{array}$ & $\underset{\substack{\infty \\
\infty}}{n}$ & 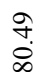 & $\stackrel{\infty}{\stackrel{\infty}{m}}$ & $\stackrel{\text { aे }}{\rightarrow}$ & $\stackrel{\infty}{0}$ \\
\hline $\begin{array}{l}\text { Penicillins (Ampicillin, Piperacilin, } \\
\text { Oxacilin, Carbenicilin, Penicillin G) }\end{array}$ & $\hat{n}$ & $\dddot{a}$ & ฉิ & $\bar{\Xi}$ & $\begin{array}{l}2 \\
i n\end{array}$ & $\begin{array}{l}\stackrel{0}{\infty} \\
\ddot{f}\end{array}$ & $\begin{array}{c}\infty \\
i \\
b\end{array}$ & $\begin{array}{l}\stackrel{0}{2} \\
\stackrel{\infty}{\infty}\end{array}$ & $\begin{array}{l}\infty \\
\infty \\
\infty\end{array}$ & 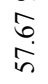 & $\stackrel{\infty}{\infty}$ & $\underset{\substack{0 \\
0}}{+}$ \\
\hline $\begin{array}{l}\text { Inhibitor-protected penicillins } \\
\text { (Piperacillin/Tazobactam, Amoxicillin } \\
\text { Clavulanic acid, Ampicillin Sulbactam, } \\
\text { Ticarcillin/ Clavulanic acid) }\end{array}$ & $\stackrel{2}{2}$ & 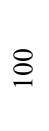 & $\infty$ & 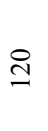 & $\underset{8}{8}$ & $\begin{array}{l}3 \\
6 \\
6\end{array}$ & $\hat{m}$ & $\stackrel{m}{m}$ & $\stackrel{7}{7}$ & $\stackrel{\overbrace{}}{\infty}$ & $\stackrel{?}{\stackrel{n}{0}}$ & : \\
\hline $\begin{array}{l}\text { Carbapenems (Imipenem, Meropenem, } \\
\text { Doripenem) }\end{array}$ & $\stackrel{m}{=}$ & 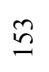 & $\Xi$ & ठิ & 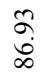 & $\frac{\infty}{\infty}$ & $\begin{array}{c}\hat{N} \\
\text { Na }\end{array}$ & $\begin{array}{l}n \\
\tilde{n} \\
\dot{a}\end{array}$ & $\stackrel{\text { ra }}{\stackrel{\sigma}{\dot{a}}}$ & $\begin{array}{l}\infty \\
\stackrel{\infty}{0} \\
\vdots \\
a\end{array}$ & $\underset{i}{\vec{j}}$ & $\stackrel{\circ}{\circ}$ \\
\hline Tetracyclines (Tetracycline) & $g$ & 8 & 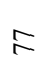 & $\curvearrowleft$ & 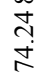 & 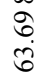 & $\underset{\substack{0 \\
\infty}}{\dot{0}}$ & $\stackrel{n}{\infty}$ & $\stackrel{s}{\stackrel{n}{\infty}}$ & 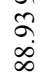 & $\underset{+}{+}$ & $\frac{\pi}{3}$ \\
\hline $\begin{array}{l}\text { Glycopeptides (Vancomycin, } \\
\text { Teicoplanin) }\end{array}$ & F & F & $\ddot{\sim}$ & $\tilde{\lambda}$ & 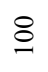 & 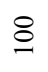 & 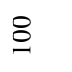 & 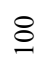 & 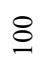 & 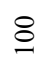 & 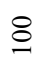 & 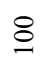 \\
\hline Oxazolidinones (Linezolid) & $\stackrel{\sim}{\sim}$ & $\stackrel{\sim}{\sim}$ & $a$ & $a$ & 8 & 8 & 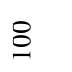 & 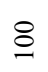 & 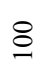 & 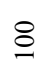 & 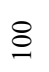 & 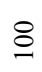 \\
\hline $\begin{array}{l}\text { Lincosamides (Lincomycin, Clindamy- } \\
\text { cin) }\end{array}$ & $\stackrel{\sim}{\sim}$ & $\approx$ & $\mathscr{0}$ & $\vec{\sim}$ & $\stackrel{\text { ָे }}{\stackrel{t}{\sim}}$ & $\begin{array}{l}\infty \\
\dot{\infty}\end{array}$ & $\underset{\substack{\infty \\
\infty}}{\curvearrowright}$ & $\frac{\partial}{\circ}$ & $\hat{a}$ & $\begin{array}{l}\text { Fे } \\
\dot{\sigma}\end{array}$ & $\stackrel{0}{?}+$ & $\underset{\substack{n \\
\infty \\
\infty}}{0}$ \\
\hline Macrolides (Azithromycin) & $\stackrel{\infty}{-1}$ & $\stackrel{\infty}{\sim}$ & 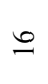 & ㄱ. & ते & in & 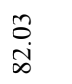 & $\stackrel{8}{\stackrel{\infty}{\infty}}$ & 广্் & $\stackrel{n}{n}$ & 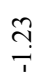 & $\tilde{\hat{n}}$ \\
\hline Amphenicols (Chloramphenicol) & $\approx$ & શे & $\stackrel{\sim}{\sim}$ & $\hat{\sim}$ & $\underset{\infty}{\tilde{D}}$ & $\begin{array}{l}: \\
\stackrel{2}{r}\end{array}$ & $\underset{\substack{\infty \\
\infty}}{\stackrel{0}{\infty}}$ & $\begin{array}{l}\infty \\
\infty \\
\infty \\
\infty\end{array}$ & 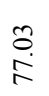 & $\stackrel{+}{\stackrel{8}{8}}$ & 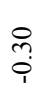 & 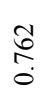 \\
\hline $\begin{array}{l}\text { Ansamycins } \\
\text { (Rifampicin) }\end{array}$ & $\underline{m}$ & $\because$ & 6 & 6 & $\begin{array}{l}\hat{6} \\
\dot{\infty}\end{array}$ & $\frac{0}{8}$ & 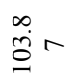 & 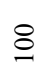 & 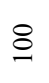 & 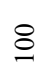 & $\stackrel{\sim}{?}$ & $\frac{n}{\mathfrak{g}}$ \\
\hline Monobactams (Aztreonam) & \pm & $\vec{\sim}$ & $r$ & $\cong$ & 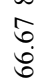 & 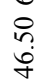 & $\begin{array}{l}3 \\
\infty \\
\infty \\
\infty \\
\infty\end{array}$ & $\stackrel{8}{\circ}$ & 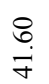 & $\begin{array}{l}\stackrel{q}{+} \\
\infty \\
\infty\end{array}$ & $\frac{9}{i}$ & $\begin{array}{l}n \\
\hat{\infty} \\
\infty \\
0\end{array}$ \\
\hline $\begin{array}{l}\text { Other antibiotics (Polymixin B, Fusidic } \\
\text { acid, Fosfomycin) }\end{array}$ & $\infty$ & $\infty$ & $\infty$ & $\hat{0}$ & $\frac{2}{\infty}$ & $\underset{\infty}{\stackrel{\infty}{\infty}}$ & \begin{tabular}{l}
$\tilde{N}$ \\
\multirow{2}{O}{}
\end{tabular} & 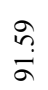 & $\underset{\infty}{m}$ & $\begin{array}{l}\infty \\
\infty \\
\infty\end{array}$ & $\stackrel{\circ}{\circ}$ & 蒡 \\
\hline $\begin{array}{l}\text { Other synthetic antibacterial agents } \\
\text { (Nitrofurantion, Trimethoprim) }\end{array}$ & $\simeq$ & $\stackrel{0}{-}$ & + & r & $\begin{array}{l}8 \\
i \\
i\end{array}$ & $\stackrel{\infty}{\stackrel{\infty}{n}}$ & $\begin{array}{l}\text { तิ } \\
\swarrow\end{array}$ & $\underset{i}{i}$ & $\stackrel{\infty}{\stackrel{\infty}{+}}$ & $\begin{array}{l}\infty \\
\infty \\
\infty\end{array}$ & $\stackrel{\infty}{\infty}$ & $\stackrel{\infty}{\underset{0}{\ddagger}}$ \\
\hline
\end{tabular}

rifampicin, $86.7 \%$ and $100 \%$, respectively; and other antiantibiotics (Polymixin B, Fusidic acid, Fosfomycin), 88\% and $91.6 \%$, respectively. Klepsiella oxytoca, Enterobacter aerogenes, Serratia marcescens, and Proteus mirabilis showed $100 \%$ susceptibility to carbapenems in both groups; Escherichia coli, Klebsiella pneumoniae, and Enterobacter cloacae were highly susceptible (more than 90\%), moderate susceptibility was detected in Pseudomonas aeruginosa, $70 \%$ in group 1 and $84.6 \%$ in group 2; Acinetobacter baumanni were more resistant, $46.2 \%$ and $40 \%$, respectively. Klebsiella pneumoniae and Klepsiella oxytoca were $100 \%$ susceptible to Chlorampenicol in both groups. Staphylococcus aureus and Acinetobacter baumanni were $100 \%$ susceptible to Rifampicin, and Streptococcus pneumonia was resistant. Staphylococcus aureus, Klepsiella oxytoca, and Enterobacter aerogenes showed $100 \%$ susceptibility to the group of other antibiotics; Acinetobacter baumanni was

highly susceptible (more than 90\%).

The following drugs are recommended by the Ministry of Health of the Republic of Kazakhstan for empiric treatment of pneumonia (community-acquired pneumonia) in adults, according to the clinical protocol (34): macrolides (64.3\% and $80 \%$, respectively), second $(65.8 \%$ and $81.1 \%$, respectively) and third generation fluoroquinolone (64.3\% and $78.3 \%$, respectively), and second generation cephalosporins $(64 \%$ and $75.7 \%$, respectively) showed moderate activity against infectious pathogens. The pathogens showed moderate susceptibility to the first generation aminoglycosides $(68.1 \%$ and $76.7 \%$, respectively), inhibitor-protected penicillins (76\% and $73.3 \%$, respectively), tetracyclines $(74.2 \%$ and $81 \%$, respectively), lincosamides $(74.3 \%$ and $76.2 \%$, respectively), monobactams $(66.7 \%$ and $70 \%$, respectively), and other synthetic antibacterial agents ( $75 \%$ and $57.1 \%$, respectively). The pathogens were less 
susceptible to penicillins ( $55.8 \%$ and $49 \%)$ in both groups.

\section{Discussion}

Our results should be considered as an unfavorable trend towards an increase in the prevalence of resistant isolates. The key reasons for this phenomenon may include high popularity and availability of these drugs in Kazakh medical practice $(35,36)$ and a fairly wide range of mutant selection windows for antimicrobial drugs from this group (37). Due to a high risk of parallel environmental damage (ie, high probability of rapid growth of resistance of other epidemically important groups of microorganisms, eg, M. TB), fluoroquinolones are considered as reserve drugs and may be used to treat pneumococcal infections in cases of resistance to other antibacterial drugs (38).

The study showed that broad-spectrum antibiotics with antituberculosis activity as well as those recommended for the treatment of nosocomial infections, showed low in vitro activity against nonspecific microorganisms in the patients with multidrug-resistant tuberculosis compared to the control group. Susceptibility to aminoglycosides (amikacin and kanamycin) was $92(77.3 \%)$ out of 119 in group $2(\mathrm{n}=106 ; 90.6 \% ; \mathrm{p}<0.05)$ and to fluoroquinolones (levofloxacin, ofloxacin) $(\mathrm{n}=74 ; 68.5 \%)$ out of 108 in group $2(n=69 ; 81.2 \%$ out of $85 ; \mathrm{p}<0.005)$. Low activity of these drugs may be due to their long-term use by the patients from group I. Therefore, microorganisms gradually developed resistance to them. 2-IV generation cephalosporins also showed low in vitro activity against nonspecific microorganisms in multidrug-resistant TB patients compared to the control group $(\mathrm{p}<0.05)$.

Klebsiella pneumoniae, isolated from pathological materials of the respiratory organs, was most often susceptible to carbapenems (imipenem, meropenem, and doripenem), $96.3 \%$ and $98.4 \%$; polymyxin B, $94.1 \%$ and $96.8 \%$; second generation aminoglycosides, $88.4 \%$ and $91.1 \%$; and third generation aminoglycosides, $84.6 \%$ and $96.4 \%$, respectively. Antibacterial drugs showed moderate activity against Pseudomonas aeruginosa and Serratia marcescens in both groups, $69.5 \%$ and $72.2 \%$, in the patients with multidrug resistance and $71.2 \%$ and $81.1 \%$ in patients with preserved drug susceptibility. No resistance was found in Staphylococcus aureus to inhibitor-protected penicillins, glycopeptides, linezolid, rifampicin, tetracycline, and a group of other antibiotics in both groups, to second and third generation aminoglycosides and cephalosporins in group 2; high activity was shown for lincosamides, $84.6 \%$ and $89 \%$, respectively; moderate activity was shown for second generation fluoroquinolones, $72 \%$ and $78.6 \%$, third generation fluoroquinolones, $83.3 \%$ and $80 \%$, and penicillins, $64.7 \%$ and $83.3 \%$, respectively.

The strains of Acinetobacter baumanni had a high resistance to aminoglycosides (48\% in group 1 and $28.6 \%$ in group 2), cephalosporins $(30 \%$ and $12.5 \%)$, fluoroquinolones $(41.7 \%$ and $20 \%)$, penicillins $(38.9 \%$ and $31.3 \%$, and carbapenems $(46.2 \%$ and $40 \%)$, respectively; and tetracycline, glycopeptides, linezolid, lincosamides, azithromycin, rifampicin, and a group of other antibiotics showed high activity. Macrolides showed moderate activity against Streptococcus pneumonia in both groups, $50 \%$ in group 1 and $60 \%$ in group 2 . Our results for the susceptibility of pneumococci to macrolides comply with the results of similar Russian studies: in 2014-2015, in Russia, 24.9\% of clinical isolates were resistant to azithromycin (39).

Our study showed an expectedly high proportion of tetracycline-resistant pneumococcal strains $(66.7 \%$ and $60 \%$, respectively), which is a consequence of a prolonged and practically uncontrolled use of these drugs in Kazakhstan. Low activity was shown by fluoroquinolones, $20 \%$ to $25 \%$ in group 1 and $50 \%$ in group 2 . The results obtained indicate that beta-lactam antibacterial drugs maintain their high activity against pneumococcal pathogens (100\% sensitivity). Taking into account that pneumococci lack enzymatic mechanisms of resistance to antibiotics of this class, the optimal drug of choice for oral therapy of respiratory infections is amoxicillin. Therefore, this drug was included in the clinical protocols of the MoHRK for the treatment of community-acquired pneumonia as a first-line therapy (29); also, the maximum sensitivity was shown by glycopeptides and linezolid.

Antibacterial drugs showed high activity against Klepsiella oxytoca, Enterobacter aerogenes, and Proteus mirabilis in both groups, $88.1 \%, 98.2 \%$, and $95.2 \%$ in patients with multidrug resistance and $92.2 \%, 94.1 \%$, and $85.7 \%$ in patients with preserved drug susceptibility. Antibacterial drugs showed moderate activity against Enterobacter cloacae in the patients with multidrug resistance $(67.5 \%)$ and high activity in the patients with preserved drug susceptibility (91.5\%). Antibacterial drugs showed low activity against Enterococcus faecalis, 21.1\% in the patients with multidrug resistance and $52.4 \%$ in patients with preserved drug susceptibility.

\section{Conclusion}

The Regional Tuberculosis Dispensary of Karaganda together with the shared-use laboratory developed, tested and implemented microbiological monitoring of the materials from the respiratory system of all patients admitted for tuberculosis treatment. The most promising groups of antibacterial drugs for the treatment of nonspecific respiratory infections in a phthisiatric clinic are vancomycin, teicoplanin, linezolid, rifampicin, carbapenems, amphenicols, second and third generation aminoglycosides, first generation fluoroquinolones, second and fourth generation cephalosporins, and a group of other antibiotics. Secondary microorganisms isolated in patients with multidrug-resistant tuberculosis are represented by the strains that are resistant to modern antibacterial drugs, and for appropriate antibiotic prescription, it is necessary to study materials from the respiratory organs in all patients admitted for tuberculosis treatment to obtain the spectrum of nonspecific microorganisms and assess their sensitivity to antimicrobial drugs.

\section{Conflict of Interests}

The authors declare that they have no competing interests. 


\section{References}

1. Global Tuberculosis Report. 2014. [cited 2020 Oct 21]. Available from:

https://apps.who.int/iris/bitstream/handle/10665/137094/97892415648 09 eng.pdf? sequence $=1$ \&isAllowed $=y$.

2. Draft global strategy and targets for tuberculosis prevention, care and control after 2015. 2014. [cited 2020 Oct 21]. Available from: http://apps.who.int/gb/ebwha/pdf files/WHA67/A67 11-en.pdf.

3. Data Global tuberculosis report. 2016 . [cited 2020 Oct 21]. Available from: http://apps.who.int/iris/bitstream/10665/250441/1/9789241565 394-eng.pdf.

4. Skak K, Nurtazina ZhB, Tabriz NS. Tuberculosis multidrug-resistant. Literature reviews. Sci Health. 2016;6:179-92.

5. Skak K, Tabriz NS, Nurtazina ZhB, Mutaikhan Zh. Drug-Resistant Tuberculosis and Modern Approaches towards its Diagnosis. Intern J Clin Skills. 2017;11(3):73-8.

6. Vishnevsky B. Drug resistance of mycobacterium tuberculosis. Lecture information. Med Alliance. 2017;1:29-35.

7. Yablonsky PK, Vishnevsky BI, Solovieva NS, Manicheva OA, Dogonadze MZ, Zhuravlev VY. Drug resistance of mycobacterium tuberculosis in different localizations of the disease. Infect Immun. 2016;2:133-40.

8. Villa-Rosas C, Laniado-Laborín R, Oceguera-Palao L. Primary drug resistance in a region with high burden of tuberculosis. A critical problem. Salud Pub Mexico. 2015;57(2):177-179.

9. Madzhuga AG, Kislyakov PA, Abdullina LB, Serdakova KG, Sadovnikova TI. Human health as a multidimensional phenomenon: Approaches to study and phenomenology. J Pharm Sci Res. 2018;10(11):2972-5.

10. Skak K, Nurtazina ZhB, Tabriz NS. Characterizition and analysis of patients with drug resistent tuberculosis. In: Materials of the XIV international research and practice conference. Sci Educ (pp. 114118). Munich, Germany, 2016.

11. Tabriz NS, Ospanova BA, Nurtazina ZhB, Skak K. Features of the course and effectiveness of treatment of patients with extensively drug-resistant tuberculosis. Mat Int Confer Integr Tubercul Control. 2016;2(28):120-1.

12. Tabriz NS, Skak K, Kassayeva LT, Omarkulov BK, Grigolashvili MA. Efficacy of the Xpert MTB/RIF Assay in Multidrug-Resistant Tuberculosis. Microb Drug Resist. 2020. [cited 2020 Oct 21]. Available from: https://www.liebertpub.com/doi/abs/10.1089/mdr. 2019.0326

13. Romashin OV, Liadov KV, Makarova MR, Koneva ES, Preobrazhenskiǐ VI, Chudimov VF. The development of physical education as a basic instrument of rehabilitative treatment, remedial medicine, and goal-oriented health promotion for the benefit of man. Vopr Kurort Fiz i Lech Fiz Kult. 2013;1:39-43.

14. Bastard M, Sanchez-Padilla E, Du Cros P, Khamraev AK, Parpieva $\mathrm{N}$, Tillyashaykov $\mathrm{M}$, et al. Outcomes of HIV-infected versus HIVnon-infected patients treated for drug-resistance tuberculosis: Multicenter cohort study. PLoS One. 2018;13(3):e0193491.

15. Rudenko SA, Mordyk AV, Bagisheva NV, Emelyanova YuA. Comparative aspects of bacteriological diagnostics, course and results of treatment of newly diagnosed tuberculosis, isolated and in combination with chronic obstructive pulmonary disease. Man Health. 2017:3:49-54

16. Gizatullina ED. Characteristics of nonspecific microflora in patients with tuberculosis. Pract Med. 2011;1(40):78-81.

17. Rachina S, Ivanchik N, Kozlov R. Features of microbiological diagnosis in community-acquired pneumonia in adults. Pract Pulmon. 2016;4:40-6.

18. Mariandyshev A, Eliseev P. Drug-resistant tuberculosis threatens WHO's End-TB strategy. Lancet Infect Diseas. 2017;17(7):674-675.

19. Variava E, Martinson N. Drug-resistant tuberculosis. Lancet Infect Dis. 2018;18(7):705-706.

20. Baugh L, Phan I, Begley DW, Clifton MC, Armour B, Dranow DM, et al. Increasing the structural coverage of tuberculosis drug targets. Tuberculosis. 2015;95(2):142-148.

21. Bezhin AI, Lipatov VA, Fronchek EV, Grigoryan AYu, Naimzada MDZ. Treatment of infected wounds with a chitosancollagen complex with dioxydin and lidocain in the experimental conditions. Med News N Caucasus. 2019;14(1):159-63.
22. Kapata N, Grobusch MP, Chongwe G, Chanda-Kapata P, Ngosa W, Tembo M, et al. Outcomes of multidrug-resistant tuberculosis in Zambia: A cohort analysis. Infection. 2017;45(6):831-839.

23. Workicho A, Kassahun W, Alemseged F. Risk factors for multidrugresistant tuberculosis among tuberculosis patients: A case-control study. Infect Drug Resist. 2017;10:91-96.

24. Yarmamedov DM, Lipatov VA, Medvedeva MV, Zaharova KV. Study of the pharmacological impact of polymeric membranes with antibacterial effect in traumatic lesions of cornea. Res Results Pharmacol. 2018;4(4):89-96.

25. Mily A, Rekha RS, Kamal SMM, Arifuzzaman ASM, Rahim Z Khan L, et al. Significant effects of oral phenylbutyrate and Vitamin D3 adjunctive therapy in pulmonary tuberculosis: A randomized controlled trial. PLoS One. 2015;10(9):e0138340.

26. Pappalardo MG, Parisi GF, Tardino L, Savasta S, Brambilla I, Marseglia GL, Licari A, Leonardi S. Measurement of nitric oxide and assessment of airway diseases in children: An update. Minerva Pediatr. 2019;71(6):524-32.

27. Falzon D, Mirzayev F, Wares F, Baena IG, Zignol M, Linh N, et al. Multidrug-resistant tuberculosis around the world: What progress has been made? Eur Respirat J. 2015;45(1):150-160.

28. Workicho A, Kassahun W, Alemseged F. Risk factors for multidrugresistant tuberculosis among tuberculosis patients: A case-control study. Infect Drug Resist. 2017;10:91-96.

29. Methodological guidelines of the Ministry of Health of Russia: Determination of the sensitivity of microorganisms to antibacterial drugs. 2012. [cited 2020 Oct 21]. Available from: https://files.stroyinf.ru/Data2/1/4293754/4293754463.pdf.

30. Spicuzza L, Parisi GF, Tardino L, Ciancio N, Nenna R, Midulla F, Leonardi S. Exhaled markers of antioxidant activity and oxidative stress in stable cystic fibrosis patients with moderate lung disease. $\mathrm{J}$ Breath Res. 2018;12(2):026010.

31. Bizzini A, Durussel C, Bille J, Greub G, Prod'hom G. Performance of matrix-assisted laser desorption ionization-time of flight mass spectrometry for identification of bacterial strains routinely isolated in a clinical microbiology laboratory. J Clin Microb. 2011;48:1549-54.

32. CLSI Publishes New Guideline: Laboratory Quality Control Based on Risk Management (EP23-A). 2011. [cited 2020 Oct 21]. Available from: https://clsi.org/media/1426/ep.23a sample.pdf.

33. Bongiovanni A, Parisi GF, Scuderi MG, Licari A, Brambilla I, Marseglia GL, Leonardi S. Gastroesophageal reflux and respiratory diseases: Does a real link exist? . Minerva Pediatr. 2019;71(6):515-23.

34. Azizov IS, Lavrinenko AV, Kolesnichenko SI. Antimicrobial susceptibility of Streptococcus pneumoniae in Kazakhstan. Clin Microb Antimicrob Chemother. 2019;21(2):187-92.

35. Clinical protocols of the Ministry of Health of the Republic of Kazakhstan. Pneumonia in adults (community-acquired pneumonia). 2017. [cited 2020 Oct 21]. Available from: https://diseases.medelement.com/disease

36. WHO Report on Surveillance of Antibiotic Consumption. 2018 [cited 2020 Oct 21]. Available from: www.who.int/medicines/areas/rational_use/who-amr-amcreport-2018 1109.pdf.

37. Ihambaeva AN, Mazhitov TM, Aldiyarova NT, Dyusembaeva NK. Dynamics and structure of consumption of systemic antimicrobial drugs. Neuros Neurol Kazakhstan. 2016;45(4):14-7.

38. Bogaevskaya O, Batrakova I, Slyusar O, Talismanov V. Pharmacogenetic testing: Effectiveness of the use of the indirect anticoagulant warfarin. J Glob Pharma Technol. 2020;12:160-9.

39. Shen GH, Tsao TC, Kao SJ. Does empirical treatment of community-acquired pneumonia with fluoroquinolones delay tuberculosis treatment and result in fluoroquinolone resistance in Mycobacterium tuberculosis? Controversies and solutions. Int $\mathrm{J}$ Antimicrob Agents. 2012;39:201-5. 